\title{
Sharp Estimates of Transition Probability Density for Bessel Process in Half-Line
}

\author{
Kamil Bogus · Jacek Małecki
}

Received: 14 October 2013 / Accepted: 5 January 2015 / Published online: 17 January 2015

(C) The Author(s) 2015. This article is published with open access at Springerlink.com

\begin{abstract}
In this paper we study the Bessel process $R_{t}^{(\mu)}$ with index $\mu \neq 0$ starting from $x>0$ and killed when it reaches a positive level $a$, where $x>a>0$. We provide sharp estimates of the transition probability density $p_{a}^{(\mu)}(t, x, y)$ for the whole range of space parameters $x, y>a$ and every $t>0$.
\end{abstract}

Keywords Transition probability density $\cdot$ Heat kernel $\cdot$ Bessel process $\cdot$ Sharp estimate Half-line

Mathematics Subject Classification (2010) $60 \mathrm{~J} 60$

\section{Introduction}

Let $R_{t}^{(\mu)}$ be the Bessel process with index $\mu \neq 0$. The transition probability density (with respect to the Lebesgue measure) of the process is expressed by the modified Bessel function in the following way

$$
p^{(\mu)}(t, x, y)=\frac{1}{t}\left(\frac{y}{x}\right)^{\mu} y \exp \left(-\frac{x^{2}+y^{2}}{2 t}\right) I_{|\mu|}\left(\frac{x y}{t}\right), \quad x, y, t>0 .
$$

Our main goal is to describe behaviour of densities of the transition probabilities for the process $R_{t}^{(\mu)}$ killed when it leaves a half-line $(a, \infty)$, where $a>0$. Note that if the process

The project was funded by the National Science Centre grant no. 2013/11/D/ST1/02622.

\footnotetext{
K. Bogus · J. Małecki $(\bowtie)$

Faculty of Fundamental Problems of Technology, Department of Mathematics,

Wrocław University of Technology, ul. Wybrzeże Wyspiańskiego 27,

50-370 Wrocław, Poland

e-mail: jacek.malecki@pwr.edu.pl

K. Bogus

e-mail: kamil.bogus@pwr.edu.pl
} 
starts from $x>a$ then the first hitting time $T_{a}^{(\mu)}$ of a level $a$ is finite a.s. when $\mu<0$ but it is infinite with positive probability when $\mu>0$. The density kernel of the killed semi-group is given by the Hunt formula

$$
p_{a}^{(\mu)}(t, x, y)=p^{(\mu)}(t, x, y)-\mathbf{E}_{x}^{(\mu)}\left[t>T_{a}^{(\mu)} ; p^{(\mu)}\left(t-T_{a}^{(\mu)}, R_{T_{a}^{(\mu)}}^{(\mu)}, y\right)\right],
$$

where $x, y>a$ and $t>0$. The main result of the paper is given in

Theorem 1 Let $\mu \neq 0$ and $a>0$. For every $x, y>a$ and $t>0$ we have

$$
p_{a}^{(\mu)}(t, x, y) \stackrel{\mu}{\approx}\left[1 \wedge \frac{(x-a)(y-a)}{t}\right]\left(1 \wedge \frac{x y}{t}\right)^{|\mu|-\frac{1}{2}}\left(\frac{y}{x}\right)^{\mu+\frac{1}{2}} \frac{1}{\sqrt{t}} \exp \left(-\frac{(x-y)^{2}}{2 t}\right) .
$$

Here $f(t, x, y) \stackrel{\mu}{\approx} g(t, x, y)$ means that there exist positive constants $c_{1}$ and $c_{2}$ depending only on the index $\mu$ such that $c_{1} \leq f / g \leq c_{2}$ for every $x, y>a$ and $t>0$. Since the constants are independent of $a>0$, one can pass to the limit with $a \rightarrow 0^{+}$and obtain the well-known estimates of $p^{(\mu)}(t, x, y)$. Since the function $I_{\mu}(z)$ behaves as a power function at zero and that some exponential term appears in the asymptotic expansion at infinity (see Preliminaries for the details), the behaviour of $p^{(\mu)}(t, x, y)$ depends on the ratio $x y / t$. Note that similar situation takes place in the case of $p_{a}^{(\mu)}(t, x, y)$, which depends on $x y / t$ as well. It can be especially seen in the proof of Theorem 1, where different methods and arguments are applied to obtain estimates (1.3), whenever $x y / t$ is large or small. Finally, taking into account the behaviour of $p^{(\mu)}(t, x, y)$, one can rewrite the statement of Theorem 1 in the following way

$$
\frac{p_{a}^{(\mu)}(t, x, y)}{p^{(\mu)}(t, x, y)} \underset{\approx}{\approx}\left(1 \wedge \frac{(x-a)(y-a)}{t}\right)\left(1 \vee \frac{t}{x y}\right), \quad x, y>a, \quad t>0,
$$

where the expression on the right-hand side of Eq. 1.4 should be read as the description of the behaviour of $p_{a}^{(\mu)}(t, x, y)$ near the boundary $a$. Since $x-a$ is just the distance of $x$ to the boundary of the considered set, we can see that the first factor of the right-hand side of Eq. 1.4 is also present in the estimates provided in [27], but the second expression is not. This is a consequence of the appearance of the different constants in the exponential terms in Thm 1.1 and Thm. 1.2 in [27]. Note also that estimates of the heat kernels of a domain are sometimes expressed in terms of survival probabilities $P_{x}(T>t)$, i.e. the probability that the process starting from $x$ does not exit the set before time $t$. For example, two-sided Gaussian type estimates for the Dirichlet heat kernel in an inner uniform domain in a Harnack-type Dirichlet space are provided in [17] (see Thm 5.16), where the considered Dirichlet kernel is shown to be bounded by the expression $P_{x}(T>t) P_{y}(T>t) p(c t, x, y)$. Here $p(t, x, y)$ stands for the global heat kernel, but in the lower and upper bounds the time $t$ is scaled by different constants. To compare this result to Eq. 1.4 recall that the survival probabilities in our case were estimated in [3] (see Eq. 2.8) and for $\mu>0$ we have

$$
\mathbf{P}_{x}^{(\mu)}\left(T_{a}^{(\mu)}>t\right) \stackrel{\mu}{\approx} \frac{x-a}{x}, \quad x<t, \quad \text { and } \quad \mathbf{P}_{x}^{(\mu)}\left(T_{a}^{(\mu)}>t\right) \stackrel{\mu}{\approx} 1 \wedge \frac{x-a}{\sqrt{t}}, \quad x \geq t .
$$

Thus, it is easy to see that

$$
p_{a}^{(\mu)}(t, x, y) \stackrel{\mu}{\approx} \mathbf{P}_{x}^{(\mu)}\left(T_{a}^{(\mu)}>t\right) \mathbf{P}_{y}^{(\mu)}\left(T_{a}^{(\mu)}>t\right) p^{(\mu)}(t, x, y), \quad x y \leq t,
$$


but we can not rewrite Eq. 1.4 in that form in the whole range of parameters, i.e. in general some additional factor appears (take for example $t<a$ and $x, y$ such that $x-a \leq \sqrt{t} \leq$ $y-a$ but $(x-a)(y-a) \geq t)$. This is one again the consequence of the fact that we do not scale the time variable in our estimates, which make them very precise, but do not allow us to annihilated the extra factors of that type by the exponential terms. The non-exponential part of Eq. 1.3 depends on the expressions $x y / t$ and $(x-a)(y-a) / t$ and consequently it can not be factorize (compare also with Thm. 5.11 in [17] or Thm. 5.7 in [26], where the estimates are given in terms of the harmonic profile), i.e. it is not comparable to $f(x, t) f(y, t) y^{2 \mu+1}$ for some function $f$.

There are several ways to define the function $p_{a}^{(\mu)}(t, x, y)$ hence our result and its applications can be considered from different points of view. It seems to be the most classical approach to define the heat kernel $p_{a}^{(\mu)}(t, x, y)$ as the fundamental solution of the heat equation $\left(\partial_{t}-L^{(\mu)}\right) u=0$ (see [15]), where $L^{(\mu)}$ is the Bessel differential operator. In the most classical case, i.e. when the operator $L^{(\mu)}$ is replaced by the classical Laplacian, the problem of finding description of the heat kernel has a very long history (see for example [26] and the references within) and goes back to 1980s and the works of E.B. Davies (see [10-13]). However, as we mentioned above, the known results for Dirichlet Laplacian on the subsets of $\mathbf{R}^{n}$ (see [27]) or in general on Riemannian manifolds (see [26] for the references) are only qualitatively sharp, i.e. the constants appearing in the exponential terms in the upper and lower estimates are different. Note that in our result these constants are the same and consequently, the exponential behaviour of the density is very precise. Such sharp estimates seems to be very rare.

Note also that the operator $L^{(\mu)}$ plays an important rôle in harmonic analysis, since it directly corresponds to Bessel-Fourier expansions (see [5-9, 24, 25] and references therein). However, since the set $(a, \infty)$ is unbounded, our consideration corresponds to the case when the spectrum is continuous. This operator on the set $(0,1)$ and the estimates of the corresponding Fourier-Bessel heat kernel were studied recently in [24] and [25], but once again the results presented there are only qualitatively sharp, i.e. the estimates are not sharp whenever $|x-y|^{2}>>t$. Another essential difference between the case of bounded sets and our case is that in the first one, we can limit our considerations to $t \leq 1$, by the application of the intrinsic ultracontractivity. However, the most interesting part of Theorem 1 (with difficult proof) seems to be when $t$ is large.

The third and our principal motivation comes from the theory of stochastic processes and the interpretation of $p_{a}^{(\mu)}(t, x, y)$ as a transition density function of the killed semi-group related to the Bessel process $R_{t}^{(\mu)}$. From this point of view, the present work is a natural continuation of the research started in [4] (see also [2]), where the integral representation of the density $q_{x, a}^{(\mu)}(t)$ of $T_{a}^{(\mu)}$ were provided together with its some asymptotics description. The sharp estimates of the density for the whole range of parameters with the explicit description of the exponential behaviour was given in [3]. For the in-depth analysis of the asymptotic behaviour of $q_{x, a}^{(\mu)}(t)$ see [18-20].

The case $\mu=0$ is excluded from our consideration and it will be addressed in the subsequent work. As it is very common in this theory, this case requires different methods and should be considered separately. In particular, some logarithmic behavior is expected whenever $x y<t$.

The paper is organized as follows. In Preliminaries we introduce some basic notation and recall properties and known results related to modified Bessel functions as well as Bessel processes, which are used in the sequel. In particular, using scaling property and absolute continuity of the Bessel processes we reduced our consideration only to the case $\mu>0$ and 
$a=1$. After that we turn to the proof of Theorem 1, which is split into two main parts, i.e. in Section 3 we provide estimates whenever $x y / t$ is large and in Section 4 we prove (1.3) for $x y / t$ small. In both cases the result is given in series of propositions.

\section{Preliminaries}

\subsection{Notation}

The constants depending on the index $\mu$ and appearing in theorems and propositions are denoted by capitals letters $C_{1}^{(\mu)}, C_{2}^{(\mu)}, \ldots$ We will denote by $c_{1}, c_{2}, \ldots$ constants appearing in the proofs and to shorten the notation we will omit the superscript ${ }^{(\mu)}$, however we will emphasize the dependence on the other variables, if such occurs.

\subsection{Modified Bessel Function}

The modified Bessel function of the first kind is defined as (see [14] 7.2.2 (12))

$$
I_{\mu}(z)=\sum_{k=0}^{\infty}\left(\frac{z}{2}\right)^{\mu+2 k} \frac{1}{k ! \Gamma(k+\mu+1)}, \quad z>0, \quad \mu>-1 .
$$

It is well-known that whenever $z$ is real the function is a positive increasing real function. Moreover, by the differentiation formula (see [14] 7.11 (20))

$$
\frac{d}{d z}\left(\frac{I_{\mu}(z)}{z^{\mu}}\right)=\frac{I_{\mu+1}(z)}{z^{\mu}}, \quad z>0
$$

and positivity of the right-hand side of Eq. 2.1 we obtain that $z \rightarrow z^{-\mu} I_{\mu}(z)$ is also increasing.

The asymptotic behavior of $I_{\mu}(z)$ at zero follows immediately from the series representation of $I_{\mu}(z)$

$$
I_{\mu}(z)=\left(\frac{z}{2}\right)^{\mu} \frac{1}{\Gamma(\mu+1)}+O\left(z^{\mu+2}\right), \quad z \rightarrow 0^{+},
$$

where the behaviour at infinity is given by (see [14] 7.13.1 (5))

$$
I_{\mu}(z) \sim \frac{e^{z}}{\sqrt{2 \pi z}}(1+O(1 / z)), \quad z \rightarrow \infty .
$$

Some parts of the proof strongly depends on the estimates of the ratio of two modified Bessel functions with different arguments. Here we recall the results of Laforgia given in Theorem 2.1 in [21]. For every $\mu>-1 / 2$ we have

$$
\frac{I_{\mu}(y)}{I_{\mu}(x)}<\left(\frac{y}{x}\right)^{\mu} e^{y-x}, \quad y \geq x>0 .
$$

Moreover, whenever $\mu \geq 1 / 2$, the lower bound of similar type holds, i.e. we have

$$
\frac{I_{\mu}(y)}{I_{\mu}(x)} \geq\left(\frac{x}{y}\right)^{\mu} e^{y-x}, \quad y \geq x>0
$$

\subsection{Bessel Processes}

In this section we introduce basic properties of Bessel processes. We follow the notation presented in [22] and [23], where we refer the reader for more details. 
We define a Bessel process $R_{t}^{(\mu)}$ with index $\mu \in \mathbf{R}$ as a linear diffusion having the half of the Bessel differential operator

$$
L^{(\mu)}=\frac{d^{2}}{d x^{2}}+\frac{2 \mu+1}{x} \frac{d}{d x}, \quad x>0,
$$

as its infinitesimal generator. Note that the state space of $R_{t}^{(\mu)}$ and the domain of $L^{(\mu)}$ depend on $\mu$ and the boundary condition at 0 (see [1] for details). To make the definition complete, we impose the killing condition at 0 , when $-1<\mu<0$ (it is when zero is a nonsingular point). Then the density of the transition probability (with respect to the Lebesgue measure) is given by Eq. 1.1. However, as it was mentioned in Introduction, the behaviour of the diffusion at zero is of no importance to the results. We write $\mathbf{P}_{x}^{(\mu)}$ and $\mathbf{E}_{x}^{(\mu)}$ for the probability law and the corresponding expected value of $R_{t}^{(\mu)}$ on the canonical path space with starting point $R_{0}^{(\mu)}=x>0$. The filtration of the coordinate process is denoted by $\mathcal{F}_{t}^{(\mu)}=\sigma\left\{R_{s}^{(\mu)}: s \leq t\right\}$. The laws of Bessel processes with different indices are absolutely continuous and the corresponding Radon-Nikodym derivative is described by

$$
\left.\frac{d \mathbf{P}_{x}^{(\mu)}}{d \mathbf{P}_{x}^{(v)}}\right|_{\mathcal{F}_{t}}=\left(\frac{w(t)}{x}\right)^{\mu-v} \exp \left(-\frac{\mu^{2}-v^{2}}{2} \int_{0}^{t} \frac{d s}{w^{2}(s)}\right),
$$

where $x>0, \mu, v \in \mathbf{R}$ and the above given formula holds $\mathbf{P}_{x}^{(v)}$-a.s on $\left\{T_{0}^{(v)}>t\right\}$. Here $T_{0}^{(\mu)}$ denotes the first hitting time of 0 by $R_{t}^{(\mu)}$.

For $x>0$ we define the first hitting of a given level $a>0$ by

$$
T_{a}^{(\mu)}=\inf \left\{t>0: R_{t}^{(\mu)}=a\right\} .
$$

Notice that for $\mu \leq 0$ we have $T_{a}^{(\mu)}<\infty$ a.s., but for $\mu>0$ the variable $T_{a}^{(\mu)}$ is infinite with positive probability. We denote by $q_{x, a}^{(\mu)}(s)$ the density function of $T_{a}^{(\mu)}$. The sharp estimates of $q_{x, a}^{(\mu)}(s)$ were obtained in [3]. We recall this result for $a=1$, which implies the result for every $a>0$, due to the scaling property of Bessel processes. More precisely, it was shown that for every $x>1$ and $t>0$ we have

$$
q_{x, 1}^{(\mu)}(s) \stackrel{\mu}{\approx}(x-1)\left(1 \wedge \frac{1}{x^{2 \mu}}\right) \frac{e^{-(x-1)^{2} /(2 t)}}{t^{3 / 2}} \frac{x^{2|\mu|-1}}{(t+x)^{|\mu|-1 / 2}}, \quad \mu \neq 0 .
$$

Note that in Theorem 2 in [3] there is a misprint in the formulation of the result and the expression $t^{|\mu|-1 / 2}+x^{|\mu|-1 / 2}$ appears instead of $(t+x)^{|\mu|-1 / 2}$, which makes a difference for $|\mu|<1 / 2$. However, the proofs provided in [3] corresponds to the correct version given above (see Lemma 4 therein). The above-given bounds imply the description of the survival probabilities (see Theorem 10 in [3])

$$
\mathbf{P}_{x}^{(\mu)}\left(\infty>T_{1}^{(\mu)}>t\right) \stackrel{\mu}{\approx} \frac{x-1}{\sqrt{x \wedge t}+x-1} \frac{1}{t^{\mu}+x^{2 \mu}}, \quad x>1, \quad t>0 .
$$

The main object of our study is the density of the transitions probabilities for the Bessel process starting from $x>a$ killed at time $T_{a}^{(\mu)}$. Taking into account the Hunt formula (1.2) 
and the fact that continuity of the paths implies $R_{T_{a}^{(\mu)}}^{(\mu)}=a$ a.s., we can represent $p_{a}^{(\mu)}(t, x, y)$ in terms of $p^{(\mu)}(t, x, y)$ and $q_{x, a}^{(\mu)}(s)$ in the following way

$$
\begin{aligned}
p_{a}^{(\mu)}(t, x, y) & =p^{(\mu)}(t, x, y)-r_{a}^{(\mu)}(t, x, y) \\
& =p^{(\mu)}(t, x, y)-\int_{0}^{t} p^{(\mu)}(t-s, a, y) q_{x, a}^{(\mu)}(s) d s .
\end{aligned}
$$

The scaling property of a Bessel process together with Eq. 2.10 imply that

$$
p_{a}^{(\mu)}(t, x, y)=\frac{1}{a} p_{1}^{(\mu)}\left(t / a^{2}, x / a, y / a\right), \quad x, y>a, \quad t>0
$$

Moreover, the absolute continuity property (2.6) applied for $\mu>0$ and $v=-\mu$ gives

$$
p_{1}^{(-\mu)}(t, x, y)=\left(\frac{x}{y}\right)^{2 \mu} p_{1}^{(\mu)}(t, x, y), \quad x, y>1, \quad t>0 .
$$

These two properties show that it is enough to prove Theorem 1 only for $a=1$ and $\mu>0$. To shorten the notation we will write $q_{x}^{(\mu)}(s)=q_{x, 1}^{(\mu)}(s)$. Since we consider the densities with respect to the Lebesgue measure (not with respect to the speed measure $m(d x)=$ $\left.2 x^{2 \mu+1} d x\right)$ the symmetry property of $p_{1}^{(\mu)}(t, x, y)$ in this case reads as follows:

$$
p_{1}^{(\mu)}(t, x, y)=\left(\frac{y}{x}\right)^{2 \mu+1} p_{1}^{(\mu)}(t, y, x), \quad x, y>1, \quad t>0 .
$$

Finally, for $\mu=1 / 2$ one can compute $p_{1}^{(\mu)}(t, x, y)$ explicitly from Eq. 2.10 , by using $I_{1 / 2}(z)=\sqrt{\frac{2}{\pi z}} \sinh (z)$ and the fact that $q_{x}^{(1 / 2)}(s)$ is a density of $1 / 2$-stable subordinator. More precisely, since

$$
\begin{aligned}
q_{x}^{(1 / 2)}(t) & =\frac{x-1}{x} \frac{1}{\sqrt{2 \pi t^{3}}} \exp \left(-\frac{(x-1)^{2}}{2 t}\right), \\
p^{(1 / 2)}(t, x, y) & =\frac{1}{\sqrt{2 \pi t}} \frac{y}{x}\left(\exp \left(-\frac{(x-y)^{2}}{2 t}\right)-\exp \left(-\frac{(x+y)^{2}}{2 t}\right)\right),
\end{aligned}
$$

we obtain

$$
\begin{aligned}
r_{1}^{(1 / 2)}(t, x, y) & =\int_{0}^{t} q_{x}^{(1 / 2)}(s) p^{(1 / 2)}(t-s, 1, y) d s \\
& =\frac{x-1}{x} \frac{y}{2 \pi}\left(H\left(t,(x-1)^{2},(y-1)^{2}\right)-H\left(t,(x-1)^{2},(y+1)^{2}\right)\right),
\end{aligned}
$$

where

$$
H(t, a, b)=\int_{0}^{t} \frac{1}{\sqrt{t-s}} \frac{1}{\sqrt{s^{3}}} \exp \left(-\frac{a}{2 s}\right) \exp \left(-\frac{b}{2(t-s)}\right) d s, \quad a, b>0 .
$$


Making the substitution $w=1 / s-1 / t$ and using formula 3.471.15 in [16] we get

$$
\begin{aligned}
H(t, a, b) & =\frac{1}{\sqrt{t}} \exp \left(-\frac{a+b}{2 t}\right) \int_{0}^{\infty} w^{-1 / 2} \exp \left(-\frac{a}{2} w-\frac{b}{2 t^{2} w}\right) d w \\
& =\sqrt{\frac{2 \pi}{t a}} \exp \left(-\frac{(\sqrt{a}+\sqrt{b})^{2}}{2 t}\right) .
\end{aligned}
$$

Hence we have

$$
r_{1}^{(1 / 2)}(t, x, y)=\frac{1}{\sqrt{2 \pi t}} \frac{y}{x}\left[\exp \left(-\frac{(x+y-2)^{2}}{2 t}\right)-\exp \left(-\frac{(x+y)^{2}}{2 t}\right)\right]
$$

which together with Eqs. 2.10 and 2.14 give

$$
p_{1}^{(1 / 2)}(t, x, y)=\frac{1}{\sqrt{2 \pi t}} \frac{y}{x}\left(\exp \left(-\frac{(x-y)^{2}}{2 t}\right)-\exp \left(-\frac{(x+y-2)^{2}}{2 t}\right)\right) .
$$

One can also obtain this formula using the relation between 3-dimensional Bessel process (i.e. with index $\mu=1 / 2$ ) and 1-dimensional Brownian motion killed when leaving a positive half-line. Note also that

$$
p_{1}^{(1 / 2)}(t, x, y) \approx\left(1 \wedge \frac{(x-1)(y-1)}{t}\right) \frac{y}{x} \frac{1}{\sqrt{t}} \exp \left(-\frac{(x-y)^{2}}{2 t}\right) .
$$

which is exactly (1.3) for $\mu=1 / 2$.

We end this section providing very useful relation between densities $q_{x}^{(\mu)}(t)$ with different indices, which once again follows from the absolute continuity property.

Lemma 1 For every $x>1$ and $t>0$ we have

$$
x^{\mu-1 / 2} q_{x}^{(\mu)}(t) \leq q_{x}^{(1 / 2)}(t) \leq x^{\nu-1 / 2} q_{x}^{(\nu)}(t),
$$

whenever $v \leq 1 / 2 \leq \mu$.

Proof The second inequality in Eq. 2.19 was given in Lemma 4 in [3]. To deal with the left-hand side of Eq. 2.19 we use Eq. 2.6 to obtain for every $\delta>0$ and $0<\varepsilon \leq \delta^{2} / 2 \wedge 1$

$$
\begin{aligned}
x^{\mu-1 / 2} \mathbf{E}_{x}^{(\mu)}[t-\varepsilon & \left.\leq T_{1}^{(\mu)} \leq t\right] \leq \mathbf{E}_{x}^{(1 / 2)}\left[t-\varepsilon \leq T_{1}^{(1 / 2)} \leq t ;\left(R_{t}\right)^{\mu-1 / 2}\right] \\
& \leq(1+\delta)^{\mu-1 / 2} \mathbf{E}_{x}^{(1 / 2)}\left[t-\varepsilon \leq T_{1}^{(1 / 2)} \leq t\right]+F_{\varepsilon}(x, t)
\end{aligned}
$$

where, by Strong Markov property

$$
\begin{aligned}
& F_{\varepsilon}(x, t)=\mathbf{E}_{x}^{(1 / 2)}\left[t-\varepsilon \leq T_{1}^{(1 / 2)} \leq t, R_{t} \geq 1+\delta ;\left(R_{t}\right)^{\mu-1 / 2}\right] \\
& =\mathbf{E}_{x}^{(1 / 2)}\left[t-\varepsilon \leq T_{1}^{(1 / 2)} \leq t ; \mathbf{E}_{1}^{(1 / 2)}\left[R_{t-T_{1}^{(1 / 2)}} \geq 1+\delta ;\left(R_{t-T_{1}^{(1 / 2)}}\right)^{\mu-1 / 2}\right]\right] \\
& =\int_{t-\varepsilon}^{t} q_{x}^{(1 / 2)}(u) \int_{1+\delta}^{\infty} y^{\mu-1 / 2} p^{(1 / 2)}(t-u, 1, y) d y d u .
\end{aligned}
$$


By Eq. 2.14, for every $r \in(0, \varepsilon)$ we have

$$
\begin{aligned}
\int_{1+\delta}^{\infty} y^{\mu-1 / 2} p^{(1 / 2)}(r, 1, y) d y & \leq \frac{1}{\sqrt{2 \pi r}} \int_{1+\delta}^{\infty} \exp \left(-\frac{(y-1)^{2}}{2 r}\right) y^{\mu+1 / 2} d y \\
& \leq \frac{1}{\sqrt{2 \pi r}} \exp \left(-\frac{\delta^{2}}{4 r}\right) \int_{1+\delta}^{\infty} \exp \left(-\frac{(y-1)^{2}}{4}\right) y^{\mu+1 / 2} d y \\
& \leq \frac{1}{\sqrt{2 \pi \varepsilon}} \exp \left(-\frac{\delta^{2}}{4 \varepsilon}\right) \int_{1+\delta}^{\infty} \exp \left(-\frac{(y-1)^{2}}{4}\right) y^{\mu+1 / 2} d y,
\end{aligned}
$$

where the last inequality follows from $\varepsilon \leq \delta^{2} / 2$. It implies that $F_{\varepsilon}(t, x) / \varepsilon$ vanishes when $\varepsilon$ goes to zero. Consequently, dividing both sides of Eq. 2.20 by $\varepsilon$ and taking a limit when $\varepsilon \rightarrow 0$, we arrive at

$$
x^{\mu-1 / 2} q_{x}^{(\mu)}(t) \leq(1+\delta)^{\mu-1 / 2} q_{x}^{(1 / 2)}(t) .
$$

Since $\delta$ was arbitrary, the proof is complete.

\section{Estimates for $x y / t$ Large}

We begin this Section with the application of the absolute continuity property of Bessel processes and the formula (2.17) which give the upper bounds for $\mu \geq 1 / 2$ and lower bounds for $v \leq 1 / 2$. These bounds are sharp whenever $x y \geq t$.

Proposition 1 Let $\mu \geq 1 / 2 \geq v>0$. For every $x, y>1$ and $t>0$ we have

$$
\left(\frac{x}{y}\right)^{\mu-\frac{1}{2}} p_{1}^{(\mu)}(t, x, y) \leq p_{1}^{(1 / 2)}(t, x, y) \leq\left(\frac{x}{y}\right)^{\nu-\frac{1}{2}} p_{1}^{(v)}(t, x, y) .
$$

Proof From the absolute continuity property (2.6) we get that for every $\mu \geq v>0$ and every Borel set $A \subset(1, \infty)$ we have

$$
\begin{aligned}
\int_{A} p_{1}^{(\mu)}(t, x, y) d y & =\frac{1}{x^{\mu-\nu}} \mathbf{E}_{x}^{(v)}\left[T_{1}^{(v)}>t, R_{t} \in A ;\left(R_{t}\right)^{\mu-v} \exp \left(-\frac{\mu^{2}-v^{2}}{2} \int_{0}^{t} \frac{d s}{R_{s}^{2}}\right)\right] \\
& \leq \frac{1}{x^{\mu-\nu}} \mathbf{E}_{x}^{(v)}\left[T_{1}^{(\nu)}>t, R_{t} \in A ;\left(R_{t}\right)^{\mu-v}\right]=\int_{A}\left(\frac{y}{x}\right)^{\mu-v} p_{1}^{(\nu)}(t, x, y) d y .
\end{aligned}
$$

Hence

$$
p_{1}^{(\mu)}(t, x, y) \leq\left(\frac{y}{x}\right)^{\mu-v} p_{1}^{(v)}(t, x, y) .
$$

Taking $\mu \geq 1 / 2$ and $v=1 / 2$ gives the left-hand side of Eq. 3.1 and taking $v \leq 1 / 2$ and $\mu=1 / 2$ gives the right-hand side of Eq. 3.1.

The absolute continuity can also be used to show the estimates for small times $t$ in a very similar way. Note that if $t<1$ then we always have $x y>t$. The proof of the main Theorem will be provided in subsequent propositions without the assumption that $t$ is bounded, but we present this simple proof to show that for $x y \geq t$ the estimates for small $t$ are just an immediate consequence of the absolute continuity of Bessel processes. 
Proposition 2 Let $\mu>0$. For every $x, y>1$ and $t \in(0,1]$ we have

$$
p_{1}^{(\mu)}(t, x, y) \stackrel{\mu}{\approx}\left(1 \wedge \frac{(x-1)(y-1)}{t}\right)\left(\frac{y}{x}\right)^{\mu+1 / 2} \frac{1}{\sqrt{t}} \exp \left(-\frac{(x-y)^{2}}{2 t}\right) .
$$

Proof Let $\mu \geq v>0$. Taking Borel set $A \subset(1, \infty)$ and $t \leq 1$ we have

$$
\int_{A} p_{1}^{(\mu)}(t ; x, y) d y=\frac{1}{x^{\mu-\nu}} \mathbf{E}_{x}^{(v)}\left[T_{1}^{(v)}>t ; R_{t} \in A ;\left(R_{t}\right)^{\mu-v} \exp \left(-\frac{\mu^{2}-v^{2}}{2} \int_{0}^{t} \frac{d s}{R_{s}^{2}}\right)\right] .
$$

Since $\inf \left\{R_{s}: s<t\right\}>1$ on $\left\{T_{1}^{(\nu)}>t\right\}$ we can write

$$
\begin{aligned}
\int_{A} p_{1}^{(\mu)}(t, x, y) d y & \geq \frac{1}{x^{\mu-\nu}} \mathbf{E}_{x}^{(v)}\left[T_{1}^{(v)}>t ; R_{t} \in A ;\left(R_{t}\right)^{\mu-v} \exp \left(-\frac{\mu^{2}-v^{2}}{2} t\right)\right] \\
& \geq \exp \left(-\frac{\mu^{2}-v^{2}}{2}\right) \int_{A}\left(\frac{y}{x}\right)^{\mu-v} p_{1}^{(v)}(t, x, y) d y
\end{aligned}
$$

Hence we get

$$
p_{1}^{(\mu)}(t, x, y) \geq \exp \left(-\frac{\mu^{2}-v^{2}}{2}\right)\left(\frac{y}{x}\right)^{\mu-v} p_{1}^{(v)}(t, x, y) .
$$

Now taking $\mu \geq 1 / 2$ and $v=1 / 2$ together with Eq. 2.17 and the result of Proposition 1 gives the proof of Eq. 3.3 for $\mu \geq 1 / 2$. Analogous argument applied for $\mu<1 / 2$ ends the proof.

Next proposition together with Proposition 1 provide the estimates for $x, y$ bounded away from 1 . Notice that if $x, y>c>1$ and $x y>t$ then

$$
\frac{(x-1)(y-1)}{t} \geq\left(1-\frac{1}{c}\right)^{2} \frac{x y}{t} \geq\left(1-\frac{1}{c}\right)^{2} .
$$

and consequently the right-hand side of Eq. 1.4 is comparable with a constant which means that $p_{1}^{(\mu)}(t, x, y)$ is comparable with $p^{(\mu)}(t, x, y)$.

Proposition 3 Let $\mu \geq 1 / 2 \geq v>0$. Then there exist constants $C_{1}^{(v)}, C_{2}^{(\mu)}>0$ and $C_{3}^{(\mu)}>1$ such that

$$
C_{1}^{(v)}\left(\frac{x}{y}\right)^{v+1 / 2} p_{1}^{(v)}(t, x, y) \leq \frac{1}{\sqrt{t}} \exp \left(-\frac{(x-y)^{2}}{t}\right) \leq C_{2}^{(\mu)}\left(\frac{x}{y}\right)^{\mu+1 / 2} p_{1}^{(\mu)}(t, x, y),
$$

whenever $x y \geq t$ and the upper bounds are valid with additional assumption $x, y>C_{3}^{(\mu)}$.

Proof Since the modified Bessel function $I_{\mu}(z)$ is positive, continuous and behaves like $(2 \pi z)^{-1 / 2} e^{z}$ at infinity (see Eq. 2.3) there exists constants $c_{1}=c_{1}(\mu)>1$ such that

$$
\frac{1}{c_{1}} \sqrt{\frac{t}{2 \pi x y}} \exp \left(\frac{x y}{t}\right) \leq I_{\mu}\left(\frac{x y}{t}\right) \leq c_{1} \sqrt{\frac{t}{2 \pi x y}} \exp \left(\frac{x y}{t}\right),
$$


whenever $x y \geq t$. Hence, taking into account general estimate $p_{1}^{(v)}(t, x, y) \leq p^{(v)}(t, x, y)$ (which is an immediate consequence of the definition (1.2)), we get

$$
p_{1}^{(v)}(t, x, y) \leq p^{(v)}(t, x, y) \stackrel{\nu}{\approx} \frac{1}{\sqrt{2 \pi}} \frac{1}{\sqrt{t}}\left(\frac{y}{x}\right)^{v+1 / 2} \exp \left(-\frac{(x-y)^{2}}{t}\right) .
$$

This ends the proof for small indices $0 \leq v \leq 1 / 2$.

Now let $\mu \geq 1 / 2$. Consequently, once again applying above given estimate of $I_{\mu}(x y / t)$ to Eq. 1.1 we arrive at

$$
\left(\frac{y}{x}\right)^{\mu-1 / 2} p^{(1 / 2)}(t, x, y) \geq p^{(\mu)}(t, x, y) \geq \frac{1}{c_{1}} \frac{1}{\sqrt{2 \pi t}}\left(\frac{y}{x}\right)^{\mu+1 / 2} \exp \left(-\frac{(x-y)^{2}}{2 t}\right), \quad x y \geq t,
$$

where the first inequality is just Eq. 3.1. Moreover, by Eq. 2.19, we have

$$
q_{x}^{(\mu)}(t) \leq \frac{q_{x}^{(1 / 2)}(t)}{x^{\mu-1 / 2}}=\frac{x-1}{x^{\mu-1 / 2}} \frac{1}{\sqrt{2 \pi} t^{3 / 2}} \exp \left(-\frac{(x-1)^{2}}{2 t}\right), \quad t>0, x>1 .
$$

and it together with left-hand side of Eqs. 3.5 and 2.16 imply

$$
\begin{aligned}
r_{1}^{(\mu)}(t, x, y) & =\int_{0}^{t} q_{x}^{(\mu)}(s) p^{(\mu)}(t-s, 1, y) d s \leq\left(\frac{y}{x}\right)^{\mu-1 / 2} \int_{0}^{t} q_{x}^{(1 / 2)}(s) p^{(1 / 2)}(t-s, 1, y) d s \\
& =\left(\frac{y}{x}\right)^{\mu-1 / 2} r_{1}^{(1 / 2)}(t, x, y) \\
& =\frac{1}{\sqrt{2 \pi t}}\left(\frac{y}{x}\right)^{\mu+1 / 2}\left(\exp \left(-\frac{(x+y-2)^{2}}{2 t}\right)-\exp \left(-\frac{(x+y)^{2}}{2 t}\right)\right) .
\end{aligned}
$$

Let $C_{3}^{(\mu)}=\left(1-\sqrt{\frac{2 c_{1}}{2 c_{1}+1}}\right)^{-1}$ and taking into account right-hand side of Eqs. 3.5 and 3.4 (with $c=C_{3}^{(\mu)}$ ) we obtain for $x, y>C_{3}^{(\mu)}$ that

$$
\begin{aligned}
\frac{r_{1}^{(\mu)}(t, x, y)}{p^{(\mu)}(t, x, y)} & \leq c_{1} \exp \left(\frac{(x-y)^{2}}{2 t}\right)\left(\exp \left(-\frac{(x+y-2)^{2}}{2 t}\right)-\exp \left(-\frac{(x+y)^{2}}{2 t}\right)\right) \\
& =c_{1}\left(\exp \left(-\frac{2(x-1)(y-1)}{t}\right)-\exp \left(-\frac{2 x y}{t}\right)\right) \\
& \leq c_{1}\left(\exp \left(-c_{2} \frac{2 x y}{t}\right)-\exp \left(-\frac{2 x y}{t}\right)\right),
\end{aligned}
$$

where

$$
c_{2}=\left(1-\frac{1}{C_{3}^{(\mu)}}\right)^{2}=\frac{2 c_{1}}{2 c_{1}+1}<1 .
$$

Taking into account the general estimate

$$
e^{-c_{2} z}-e^{-z} \leq \frac{1-c_{2}}{c_{2}}, \quad z>0, c_{2}<1
$$

we arrive at

$$
\frac{r_{1}^{(\mu)}(t, x, y)}{p^{(\mu)}(t, x, y)} \leq c_{1} \frac{1-c_{2}}{c_{2}}=\frac{1}{2}
$$


Consequently, using Eq. 3.5

$$
p_{1}^{(\mu)}(t, x, y) \geq \frac{1}{2} p^{(\mu)}(t, x, y) \geq \frac{1}{2 c_{1}} \frac{1}{\sqrt{2 \pi t}}\left(\frac{y}{x}\right)^{\mu+1 / 2} \exp \left(-\frac{(x-y)^{2}}{2 t}\right) .
$$

Now we turn our attention to the case when $x$ and $y$ are bounded. The next proposition, however, is much more general.

Proposition 4 For fixed $m>0$ and $\mu \geq 1 / 2 \geq v>0$ there exist constants $C_{4}^{(\mu)}, C_{4}^{(v)}>0$ such that

$$
C_{4}^{(\mu)}\left(\frac{x}{y}\right)^{\mu+1 / 2} p_{1}^{(\mu)}(t, x, y) \geq\left(1 \wedge \frac{(x-1)(y-1)}{t}\right) \frac{1}{\sqrt{t}} \exp \left(-\frac{(x-y)^{2}}{2 t}\right)
$$

and

$$
\left(1 \wedge \frac{(x-1)(y-1)}{t}\right) \frac{1}{\sqrt{t}} \exp \left(-\frac{(x-y)^{2}}{2 t}\right) \geq C_{4}^{(v)}\left(\frac{x}{y}\right)^{v+1 / 2} p_{1}^{(v)}(t, x, y)
$$

whenever $(x \wedge y)^{2} \geq m t$.

Proof By Eq. 2.12, without lost of generality, we can assume that $1<x<y$. We put $b=(x+1) / 2$ and take $\mu \geq 1 / 2$. Using Eq. 2.6 and the fact that $T_{b}^{(1 / 2)} \leq T_{1}^{(1 / 2)}$ we can write for every Borel set $A \subset(1, \infty)$ that

$$
\int_{A} p_{1}^{(\mu)}(t, x, y) d y \geq \mathbf{E}_{x}^{(1 / 2)}\left[t<T_{b}^{(1 / 2)}, R_{t} \in A ;\left(\frac{R_{t}}{x}\right)^{\mu-1 / 2} \exp \left(-\frac{\mu^{2}-1 / 4}{2} \int_{0}^{t} \frac{d s}{R_{s}^{2}}\right)\right]
$$

Since up to time $T_{b}^{(1 / 2)}$ we have

$$
\int_{0}^{t} \frac{d s}{R_{s}^{2}} \leq \frac{4 t}{(x+1)^{2}} \leq \frac{4 t}{x^{2}} \leq \frac{4}{m}
$$

we obtain

$$
\int_{A} p_{1}^{(\mu)}(t, x, y) d y \geq \exp \left(-\frac{4 \mu^{2}-1}{2 m}\right) \mathbf{E}_{x}^{(1 / 2)}\left[t<T_{b}^{(1 / 2)}, R_{t} \in A ;\left(\frac{R_{t}}{x}\right)^{\mu-1 / 2}\right],
$$

which gives

$$
p_{1}^{(\mu)}(t, x, y) \geq \exp \left(-\frac{4 \mu^{2}-1}{2 m}\right)\left(\frac{y}{x}\right)^{\mu-1 / 2} p_{b}^{(1 / 2)}(t, x, y) .
$$

From the other side, the scaling property (2.11) and the formula (2.18) give

$$
\begin{aligned}
p_{b}^{(1 / 2)}(t, x, y) & =\frac{1}{b} p_{1}^{(1 / 2)}\left(\frac{t}{b^{2}} ; \frac{x}{b}, \frac{y}{b}\right) \\
& \approx \frac{1}{\sqrt{t}} \frac{y}{x} \exp \left(-\frac{(x-y)^{2}}{2 t}\right)\left(1 \wedge \frac{(x-b)(y-b)}{t}\right) \\
& \approx \frac{1}{\sqrt{t}} \frac{y}{x} \exp \left(-\frac{(x-y)^{2}}{2 t}\right)\left(1 \wedge \frac{(x-1)(y-1)}{t}\right),
\end{aligned}
$$


where the last equalities follows from

$$
x-b=\frac{x-1}{2}, \quad \frac{y-1}{2} \leq y-b \leq y-1 .
$$

It ends the proof for $\mu \geq 1 / 2$.

For $1 / 2 \geq v>0$ and $x>2$, using the asymptotic expansion (2.3), the desired inequality just follows from

$$
p_{1}^{(v)}(t, x, y) \leq p^{(v)}(t, x, y) \stackrel{m, v}{\approx} \frac{1}{\sqrt{t}}\left(\frac{y}{x}\right)^{v+1 / 2} \exp \left(-\frac{(x-y)^{2}}{2 t}\right),
$$

which holds since $x y \geq x^{2}>m t$. To get the result note that $1 \wedge(x-1)(y-1) / t$ is comparable with constant, whenever $x$ and $y$ are bounded away from 1 and $x y>m t$. If $x \leq 2$, then $t \leq x^{2} / m \leq 4 / m$ and consequently the same arguments as in Proposition 2 give that for every Borel set $A \subset(1, \infty)$ we have

$$
\begin{aligned}
\int_{A} p_{1}^{(v)}(t, x, y) d y & =\mathbf{E}_{x}^{(1 / 2)}\left[t<T_{1}^{(1 / 2)}, R_{t} \in A ;\left(\frac{R_{t}}{x}\right)^{v-1 / 2} \exp \left(\frac{1 / 4-v^{2}}{2} \int_{0}^{t} \frac{d s}{R_{s}^{2}}\right)\right] \\
& \leq \exp \left(\frac{1-4 v^{2}}{2 m}\right) \mathbf{E}_{x}^{(1 / 2)}\left[t<T_{1}^{(1 / 2)}, R_{t} \in A ;\left(\frac{R_{t}}{x}\right)^{v-1 / 2}\right]
\end{aligned}
$$

and we obtain

$$
p_{1}^{(v)}(t, x, y) \leq \exp \left(\frac{1-4 v^{2}}{2 m}\right)\left(\frac{y}{x}\right)^{\nu-1 / 2} p_{1}^{(1 / 2)}(t, x, y) .
$$

This together with the estimates for $p_{1}^{(1 / 2)}(t, x, y)$ finish the proof.

Since for $x, y<C$ and $x y \geq t$, for some fixed $C>1$, we have

$$
\frac{(x \wedge y)^{2}}{t} \geq \frac{x y}{C t} \geq \frac{1}{C},
$$

applying the results of Proposition 4 (with $m=C^{-1}$ ) and Proposition 1 gives

Corollary 1 For every $C>1$ we have

$$
p_{1}^{(\mu)}(t, x, y) \stackrel{\mu, C}{\approx}\left(1 \wedge \frac{(x-1)(y-1)}{t}\right)\left(\frac{y}{x}\right)^{\mu+1 / 2} \frac{1}{\sqrt{t}} \exp \left(-\frac{(x-y)^{2}}{2 t}\right)
$$

whenever $x, y<C$ and $x y \geq t$.

Finally, we end this section with two propositions related to the case when one of the space variables is close to 1 and the other is large. We deal with this case separately for $\mu<1 / 2$ and $\mu \geq 1 / 2$.

Proposition 5 For every $v \in(0,1 / 2)$ there exists constant $C_{5}^{(v)}>0$ such that

$$
p_{1}^{(v)}(t, x, y) \leq C_{5}^{(v)} \frac{1}{\sqrt{t}}\left(\frac{y}{x}\right)^{v+1 / 2} \exp \left(-\frac{(x-y)^{2}}{2 t}\right)\left(1 \wedge \frac{(x-1)(y-1)}{t}\right)
$$

for $1<x \leq 2 \leq y$ and $x y \geq t$. 
Proof Using the right-hand side of Eq. 2.19, we get

$$
q_{x}^{(v)}(s) \geq \frac{x-1}{\sqrt{2 \pi s^{3}}} \frac{1}{x^{v+1 / 2}} \exp \left(-\frac{(x-1)^{2}}{2 s}\right), \quad 0 \leq v<1 / 2, s>0 .
$$

On the other side by monotonicity of $I_{v}(z)$, for every $s \in(0, t)$ we have

$$
I_{v}\left(\frac{y}{t-s}\right) \geq I_{v}\left(\frac{y}{t}\right) .
$$

Hence, combining together above-given inequalities and the formula (1.1), we obtain

$$
\begin{aligned}
r_{1}^{(v)}(t, x, y) & =\int_{0}^{t} q_{x}^{(v)}(s) \frac{y^{1+v}}{t-s} \exp \left(-\frac{1+y^{2}}{2(t-s)}\right) I_{v}\left(\frac{y}{t-s}\right) d s \\
& \geq \int_{0}^{t} q_{x}^{(v)}(s) \frac{y^{1+v}}{\sqrt{t-s}} \frac{1}{\sqrt{t}} \exp \left(-\frac{1+y^{2}}{2(t-s)}\right) I_{v}\left(\frac{y}{t}\right) d s \\
& \geq \frac{x-1}{\sqrt{2 \pi}}\left(\frac{y}{x}\right)^{v+1} \sqrt{\frac{x}{t}} I_{\nu}\left(\frac{y}{t}\right) H\left(t,(x-1)^{2}, 1+y^{2}\right) \\
& =\frac{\sqrt{x}}{t}\left(\frac{y}{x}\right)^{v+1} I_{v}\left(\frac{y}{t}\right) \exp \left(-\frac{\left(x-1+\sqrt{y^{2}+1}\right)^{2}}{2 t}\right),
\end{aligned}
$$

where the last equality follows from Eq. 2.15. Using Eq. 2.4 we obtain

$$
\begin{aligned}
p^{(v)}(t, x, y) & =\frac{y^{v+1}}{t} \exp \left(-\frac{x^{2}+y^{2}}{2 t}\right) \frac{1}{x^{v}} I_{v}\left(\frac{x y}{t}\right) \\
& \leq \frac{y^{v+1}}{t} \exp \left(-\frac{(x-y)^{2}}{2 t}\right) \exp \left(-\frac{y}{t}\right) I_{v}\left(\frac{y}{t}\right),
\end{aligned}
$$

which together with previously given estimates, Eq. 2.9 and finally Eq. 2.3 give

$$
\begin{aligned}
p_{1}^{(v)}(t, x, y) & \leq \frac{y^{\nu+1}}{t} \exp \left(-\frac{(x-y)^{2}}{2 t}\right) \exp \left(-\frac{y}{t}\right) I_{\nu}\left(\frac{y}{t}\right) f_{y, t}(x) \\
& \leq c_{1} \frac{y^{v+1 / 2}}{\sqrt{t}} \exp \left(-\frac{(x-y)^{2}}{2 t}\right) f_{y, t}(x),
\end{aligned}
$$

where

$$
f_{y, t}(x)=1-\frac{1}{x^{\nu+1 / 2}} \exp \left(-\frac{(x-1)\left(\sqrt{y^{2}+1}+y-1\right)}{t}\right) .
$$

By elementary computation we can see that

$$
\begin{aligned}
-f_{y, t}^{\prime}(x) & =\frac{1}{x^{v+3 / 2}} \exp \left(-\frac{(x-1)\left(\sqrt{y^{2}+1}+y-1\right)}{t}\right)\left(\frac{\sqrt{y^{2}+1}+y-1}{t} x+v+1 / 2\right) \\
& \leq \frac{1}{x^{v+3 / 2}}\left(\frac{2 x y}{t}+1\right) \leq \frac{4 x y}{t} \leq 16 \frac{y-1}{t} .
\end{aligned}
$$

Here we have used the following inequalities

$$
\sqrt{y^{2}+1}+y-1<2 y, \quad x y \geq t, \quad 1<x \leq 2 \leq y .
$$


Thus, by the mean value theorem, there exists $d=d_{x, y, t} \in(1, x)$ such that

$$
f_{y, t}(x)=(1-x) f_{y, t}^{\prime}(d) \leq 16 \frac{(x-1)(y-1)}{t} .
$$

Hence, combining Eqs. 3.7 with 3.8 and $f_{y, t}(x) \leq 1$, we obtain finally

$$
\begin{aligned}
p_{1}^{(v)}(t, x, y) & \leq 16 c_{1}\left(1 \wedge \frac{(x-1)(y-1)}{t}\right) \frac{y^{v+1 / 2}}{\sqrt{t}} \exp \left(-\frac{(x-y)^{2}}{2 t}\right) \\
& \leq c_{1} 2^{v+9 / 2}\left(1 \wedge \frac{(x-1)(y-1)}{t}\right)\left(\frac{y}{x}\right)^{v+1 / 2} \frac{1}{\sqrt{t}} \exp \left(-\frac{(x-y)^{2}}{2 t}\right),
\end{aligned}
$$

where the last inequality follows from $16 x^{v+1 / 2} \leq 2^{v+9 / 2}$ whenever $x \leq 2$.

Proposition 6 For every $\mu \geq 1 / 2$ and $c>1$ there exists constant $C_{6}^{(\mu)}(c)>0$ such that for every $1<x \leq c$ and $y \geq 5 c(\mu+1)$ we have

$$
p_{1}^{(\mu)}(t, x, y) \geq C_{6}^{(\mu)}(c) \frac{1}{\sqrt{t}}\left(\frac{y}{x}\right)^{\mu+1 / 2} \exp \left(-\frac{(x-y)^{2}}{2 t}\right)\left(1 \wedge \frac{(x-1)(y-1)}{t}\right),
$$

whenever $x y \geq t$.

Proof Let us fix $\mu \geq 1 / 2$. For every $0<s<t$, using Eq. 2.4, we have

$$
I_{\mu}\left(\frac{y}{t-s}\right)<I_{\mu}\left(\frac{y}{t}\right)\left(\frac{t}{t-s}\right)^{\mu} \exp \left(\frac{y}{t-s}\right) \exp \left(-\frac{y}{t}\right)
$$

and consequently

$$
\frac{p^{(\mu)}(t-s, 1, y)}{p^{(\mu)}(t, 1, y)}<\left(\frac{t}{t-s}\right)^{\mu+1} \exp \left(-\frac{(y-1)^{2}}{2}\left(\frac{1}{t-s}-\frac{1}{t}\right)\right)=\frac{g_{y}(t-s)}{g_{y}(t)},
$$

where

$$
g_{y}(w)=\left(\frac{1}{w}\right)^{\mu+1} \exp \left(-\frac{(y-1)^{2}}{2 w}\right), \quad w>0 .
$$

Note that

$$
g_{y}^{\prime}(w)=\left(\frac{1}{w}\right)^{\mu+2} \exp \left(-\frac{(y-1)^{2}}{2 w}\right)\left(\frac{(y-1)^{2}}{2 w}-(\mu+1)\right) .
$$

Since $x \leq c, y \geq 5 c(\mu+1)>2$ and $x y \geq t$ we have $4(y-1) \geq 2 y \geq 2 t / c$. Moreover $y-1 \geq 4 c(\mu+1)$. Thus

$$
\frac{(y-1)^{2}}{2 t} \geq \frac{4 c(\mu+1)(y-1)}{2 t} \geq \mu+1 .
$$

It means that under our assumptions on $x, y$ and $t$ the function $g_{y}(w)$ is increasing on $(0, t)$ and consequently $g_{y}(t-s) \leq g_{y}(t)$ for every $0<s<t$. Thus, it enable us to write

$$
\begin{aligned}
r_{1}^{(\mu)}(t, x, y) & =\int_{0}^{t} q_{x}^{(\mu)}(s) p^{(\mu)}(t-s, 1, y) d s \leq p^{(\mu)}(t, 1, y) \int_{0}^{t} q_{x}^{(\mu)}(s) d s \\
& \leq x^{-\mu} \exp \left(\frac{x^{2}-1}{2 t}\right) \frac{I_{\mu}(y / t)}{I_{\mu}(x y / t)} p^{(\mu)}(t, x, y),
\end{aligned}
$$


where the last inequality comes from fact that $\int_{0}^{t} q_{x}^{(\mu)}(s) d s \leq \int_{0}^{\infty} q_{x}^{(\mu)}(s) d s=x^{-2 \mu}$. The above-given ratio of modified Bessel functions can be estimated from above by using Eq. 2.5 as follows

$$
I_{\mu}\left(\frac{y}{t}\right) \leq I_{\mu}\left(\frac{x y}{t}\right) \exp \left(-\frac{(x-1) y}{t}\right) x^{\mu}
$$

Consequently

$$
r_{1}^{(\mu)}(t, x, y) \leq p^{(\mu)}(t, x, y) \exp \left(-\frac{(x-1)(2 y-x-1)}{2 t}\right) .
$$

Finally observe that $2 y-x-1>y-1$ and we arrive at

$$
\begin{aligned}
p_{1}^{(\mu)}(t, x, y) & \geq\left(1-\exp \left(-\frac{(x-1)(y-1)}{2 t}\right)\right) p^{(\mu)}(t, x, y) \\
& \stackrel{\mu}{\approx}\left(1 \wedge \frac{(x-1)(y-1)}{t}\right) \frac{1}{\sqrt{t}}\left(\frac{y}{x}\right)^{\mu+1 / 2} \exp \left(-\frac{(x-y)^{2}}{2 t}\right) .
\end{aligned}
$$

This ends the proof.

The proof of Eq. 1.3 in the case $x y \geq t$ can be deduced from above-given propositions in the following way. Let $\mu \geq 1 / 2$ and without any loss of generality we assume that $x \leq y$. The upper bounds for every $x, y>1$ are given in Proposition 1. From Proposition 3 we know that the lower bounds are valid for $x, y>C_{3}^{(\mu)}$. If $x \leq C_{3}^{(\mu)}$ and $y \geq 5 C_{3}^{(\mu)}(\mu+1)$ then the lower bounds are given in Proposition 6. Finally, taking $C=5 C_{3}^{(\mu)}(\mu+1)$ in Corollary 1 we get the lower bounds in the remaining range of the parameters $x$ and $y$. The proof for $v \leq 1 / 2$ is obtained in the same way.

\section{Estimates for $x y / t$ Small}

In this section we provide estimates of $p_{1}^{(\mu)}(t, x, y)$ whenever $x y<t$. Note also that Eq. 1.3 can be written in the following shorter way

$$
p_{1}^{(\mu)}(t, x, y) \stackrel{\mu}{\approx} \frac{x-1}{x} \frac{y-1}{y}\left(\frac{y^{2}}{t}\right)^{\mu+1 / 2} \frac{1}{\sqrt{t}} \exp \left(-\frac{x^{2}+y^{2}}{2 t}\right),
$$

whenever $x y<t$. The main difficulty is to obtain the estimates when one of the space parameters is close to 1 and the other is large, i.e. tends to infinity. In this case we have to take care of cancellations of two quantities appearing in Eq. 2.9 but also not to lose a control on the exponential behaviour. We begin with the upper bounds.

Proposition 7 For every $\mu>0$, there exists constant $C_{7}^{(\mu)}>0$ such that

$$
p_{1}^{(\mu)}(t, x, y) \leq C_{7}^{(\mu)} \frac{x-1}{x} \frac{y-1}{y}\left(\frac{y^{2}}{t}\right)^{\mu+1 / 2} \frac{1}{\sqrt{t}} \exp \left(-\frac{x^{2}+y^{2}}{2 t}\right)
$$

whenever $x y \leq t$. 
Proof If $x, y>2$ the result follows immediately from the general estimate $p_{1}^{(\mu)}(t, x, y) \leq$ $p^{(\mu)}(t, x, y)$ and Eq. 2.2 which gives

$$
p^{(\mu)}(t, x, y) \approx\left(\frac{y^{2}}{t}\right)^{\mu+1 / 2} \frac{1}{\sqrt{t}} \exp \left(-\frac{x^{2}+y^{2}}{2 t}\right), \quad \frac{x y}{t} \leq 1 .
$$

Note that for every $x, y>0$ and $t>0$ there exists $c_{1}>0$ such that

$$
p^{(\mu)}(t, x, y) \leq c_{1} \frac{y^{2 \mu+1}}{t^{\mu+1}} .
$$

If $x y<t$, then it immediately follows from Eq. 4.1 by estimating the exponential term by 1 . For $x y \geq t$ we use the asymptotic behaviour (2.3) to show that

$$
p^{(\mu)}(t, x, y) \approx \frac{1}{\sqrt{t}}\left(\frac{y}{x}\right)^{\mu+1 / 2} \exp \left(-\frac{|x-y|^{2}}{2 t}\right) \leq \frac{y^{2 \mu+1}}{t^{\mu+1}}\left(\frac{t}{x y}\right)^{\mu+1 / 2} \leq \frac{y^{2 \mu+1}}{t^{\mu+1}}
$$

In particular, for all $z, w>1$ and $1<y<2$ there exists $c_{2}>0$ such that

$$
p^{(\mu)}(t / 3, z, w) \leq c_{2}\left(\frac{w}{y}\right)^{2 \mu+1} \frac{1}{t^{\mu+1}} .
$$

The Chapman-Kolmogorov equation and estimating the middle term using Eq. 4.4 give

$$
\begin{aligned}
p_{1}^{(\mu)}(t, x, y) & =\int_{1}^{\infty} \int_{1}^{\infty} p_{1}^{(\mu)}(t / 3, x, z) p_{1}^{(\mu)}(t / 3, z, w) p_{1}^{(\mu)}(t / 3, w, y) d z d w \\
& \leq \frac{c_{3}}{t^{\mu+1}} \int_{1}^{\infty} p_{1}^{(\mu)}(t / 3, x, z) d z \int_{1}^{\infty}\left(\frac{w}{y}\right)^{2 \mu+1} p_{1}^{(\mu)}(t / 3, w, y) d w \\
& =\frac{c_{3}}{t^{\mu+1}} P_{x}^{(\mu)}\left(T_{1}^{(\mu)}>t / 3\right) P_{y}^{(\mu)}\left(T_{1}^{(\mu)}>t / 3\right) .
\end{aligned}
$$

Here the last equality follows from the symmetry property (2.12). Since, by Eq. 2.8 and the fact that $\int_{0}^{\infty} q_{x}^{(\mu)}(u) d u=x^{-2 \mu}$, whenever $x y<t$ and $1<x, y<2$ we have

$$
P_{x}^{(\mu)}\left(T_{1}^{(\mu)}>t / 3\right)=P_{x}^{(\mu)}\left(\infty>T_{1}^{(\mu)}>t / 3\right)+P_{x}^{(\mu)}\left(T_{1}^{(\mu)}=\infty\right) \approx \frac{x-1}{t^{\mu}}+1-\frac{1}{x^{2 \mu}} \approx x-1,
$$

which ends the proof of the upper-bound in this case.

Now assume that $y \geq 2,1<x \leq 2$ and $x y \leq t$. The other case $x \geq 2,1<y \leq$ 2 follows from the symmetry condition mentioned above. Using once again the fact that $\int_{0}^{\infty} q_{x}^{(\mu)}(u) d u=x^{-2 \mu}$ and Eq. 2.9, we can write

$$
\begin{aligned}
p_{1}^{(\mu)}(t, x, y) & \leq p^{(\mu)}(t, x, y)-\int_{0}^{1 / 2} q_{x}^{(\mu)}(u) p^{(\mu)}(t-u, 1, y) d u \\
& =J_{1}(t, x, y)+J_{2}(t, x, y)+J_{3}(t, x, y),
\end{aligned}
$$

where

$$
\begin{aligned}
& J_{1}(t, x, y)=p^{(\mu)}(t, x, y)-\frac{1}{x^{2 \mu}} p^{(\mu)}(t, x, y)+\mathbf{P}_{x}^{(\mu)}\left(\infty>T_{1}^{(\mu)}>1 / 2\right) p^{(\mu)}(t, x, y), \\
& J_{2}(t, x, y)=\mathbf{P}_{x}^{(\mu)}\left(T_{1}^{(\mu)} \leq 1 / 2\right)\left(p^{(\mu)}(t, x, y)-p^{(\mu)}(t, 1, y)\right), \\
& J_{3}(t, x, y)=\int_{0}^{1 / 2} q_{x}^{(\mu)}(u)\left(p^{(\mu)}(t, 1, y)-p^{(\mu)}(t-u, 1, y)\right), d u .
\end{aligned}
$$


It is obvious that for $1<x<2$ we have

$$
J_{1}(t, x, y) \leq c_{4}(x-1) p^{(\mu)}(t, x, y) .
$$

To deal with $J_{2}(t, x, y)$ note that the differentiation formula (2.1), the asymptotic behavior (2.2) and positivity of $I_{\mu}(z)$ give

$$
\begin{aligned}
\frac{d}{d x}\left[e^{-x^{2} / 2 t}\left(\frac{t}{x y}\right)^{\mu} I_{\mu}\left(\frac{x y}{t}\right)\right]= & -\frac{x}{t} e^{-x^{2} / 2 t}\left(\frac{t}{x y}\right)^{\mu} I_{\mu}\left(\frac{x y}{t}\right) \\
& +e^{-x^{2} / 2 t} \frac{y}{t}\left(\frac{t}{x y}\right)^{\mu} I_{\mu+1}\left(\frac{x y}{t}\right) \\
\leq & c_{5} e^{-x^{2} / 2 t}\left(\frac{x y}{t}\right)^{2} \leq c_{5},
\end{aligned}
$$

whenever $x y<t$. Consequently, by mean value theorem, we obtain

$$
J_{2}(t, x, y) \leq\left(p^{(\mu)}(t, x, y)-p^{(\mu)}(t, 1, y)\right) \leq c_{5}(x-1)\left(\frac{y^{2}}{t}\right)^{\mu+1 / 2} \frac{1}{\sqrt{t}} e^{-y^{2} / 2 t} .
$$

Finally, the bounds of $J_{3}(t, x, y)$ follow from the estimates for the derivative of $p^{(\mu)}(t, 1, y)$ in $t$. Using once again Eq. 2.1 and skipping the negative components we have

$$
\begin{aligned}
h(t, y) & \stackrel{\text { def }}{=} \frac{d}{d t}\left(\frac{1}{t^{\mu+1}} e^{-\frac{1+y^{2}}{2 t}}\left(\frac{t}{y}\right)^{\mu} I_{\mu}\left(\frac{y}{t}\right)\right) \\
& =e^{-\left(1+y^{2}\right) /(2 t)} \frac{I_{\mu}(y / t)}{t y^{\mu}}\left(-\frac{\mu+1}{t}+\frac{1+y^{2}}{2 t^{2}}-\frac{y}{t} \frac{I_{\mu+1}(y / t)}{I_{\mu}(y / t)}\right) \\
& \leq e^{-\left(1+y^{2}\right) /(2 t)} \frac{I_{\mu}(y / t)}{t y^{\mu}} \frac{1+y^{2}}{2 t^{2}} \leq c_{6} e^{-\left(1+y^{2}\right) /(2 t)} \frac{1}{t^{\mu+1}},
\end{aligned}
$$

whenever $y<t$. Thus, there exists $c=c_{\mu, u, y} \in(t-u, t)$ such that

$$
\begin{aligned}
J_{3}(t, x, y) & =\int_{0}^{1 / 2} q_{x}^{(\mu)}(u) u y^{2 \mu+1} h\left(c_{\mu, u, y}, y\right) d u \\
& \leq c_{6} y^{2 \mu+1} \int_{0}^{1 / 2} q_{x}^{(\mu)}(u) u e^{-\left(1+y^{2}\right) /(2 c)} \frac{1}{c^{\mu+1}} d u \\
& \leq c_{6} e^{-\left(1+y^{2}\right) /(2 t)} \frac{y^{2 \mu+1}}{(t / 2)^{\mu+1}} \int_{0}^{1 / 2} u q_{x}^{(\mu)}(u) d u .
\end{aligned}
$$

Taking into account the upper bounds given in Eq. 2.7 we get

$$
\int_{0}^{1 / 2} u q_{x}^{(\mu)}(u) d u \leq c_{7} \frac{x-1}{x^{\mu+1 / 2}} \int_{0}^{1 / 2} e^{-(x-1)^{2} /(2 u)} \frac{d u}{u^{1 / 2}} \leq c_{8}(x-1) .
$$

This ends the proof.

The proof of the lower bounds is split into two parts. Next proposition corresponds to the case when $y>x>1$ and $(y-1)^{2} / t$ is large. Moreover, we enlarge the region and assume that $x y<m t$ for a given $m \geq 1$. It is forced by the lower bounds given in Proposition 6 , where it is required to have $x y / t$ sufficiently large but also by the proof of Proposition 9 . 
Proposition 8 For every $\mu>0$ and $m \geq 1$, there exists constant $C_{8}^{(\mu)}(m)>0$ such that

$$
\frac{p_{1}^{(\mu)}(t, x, y)}{p^{(\mu)}(t, x, y)} \geq C_{8}^{(\mu)}(m) \frac{x-1}{x}, \quad y>x>1,
$$

whenever $x y<m t$ and $\frac{(y-1)^{2}}{t} \geq 2(\mu+1)$.

Proof Using Eq. 2.4 we have

$$
\begin{aligned}
\frac{r_{1}^{(\mu)}(t, x, y)}{p^{(\mu)}(t, 1, y)} & =\int_{0}^{t} q_{x}^{(\mu)}(s) \frac{p^{(\mu)}(t-s, 1, y)}{p^{(\mu)}(t, 1, y)} d s \\
& =\int_{0}^{t} q_{x}^{(\mu)}(s) \frac{t}{t-s} \exp \left(-\frac{1+y^{2}}{2 t} \frac{s}{t-s}\right) \frac{I_{\mu}(y /(t-s))}{I_{\mu}(y / t)} d s \\
& \leq \int_{0}^{t} q_{x}^{(\mu)}(s)\left(\frac{t}{t-s}\right)^{\mu+1} \exp \left(-\frac{(y-1)^{2}}{2 t} \frac{s}{t-s}\right) d s .
\end{aligned}
$$

Now, for every $s<t$ we can write

$$
\left(\frac{t}{t-s}\right)^{\mu+1} \exp \left(-\frac{(y-1)^{2}}{2 t} \frac{s}{t-s}\right)=\frac{f_{y}(t-s)}{f_{y}(t)},
$$

where $f_{y}(w)=w^{-\mu-1} e^{-(y-1)^{2} / 2 w}$. Then by simple calculation we get $f_{y}^{\prime}(w)=$ $w^{-\mu-2} e^{-(y-1)^{2} / 2 w}\left(\frac{(y-1)^{2}}{2 w}-(\mu+1)\right)$ and consequently $f_{y}(w)$ is increasing on $\left(0, \frac{(y-1)^{2}}{2(\mu+1)}\right)$. It implies that right-hand side of Eq. 4.5 is smaller than 1 whenever $\frac{(y-1)^{2}}{t} \geq 2(\mu+1)$ and as consequence we obtain

$$
\frac{r_{1}^{(\mu)}(t, x, y)}{p^{(\mu)}(t, 1, y)} \leq \int_{0}^{t} q_{x}^{(\mu)}(s) d s
$$

Now notice that

$$
\frac{p_{1}^{(\mu)}(t, x, y)}{p^{(\mu)}(t, x, y)}=1-\frac{p^{(\mu)}(t, 1, y)}{p^{(\mu)}(t, x, y)} \frac{r_{1}^{(\mu)}(t, x, y)}{p^{(\mu)}(t, 1, y)},
$$

where from Eqs. 4.6 and 1.1 we get

$$
\frac{p_{1}^{(\mu)}(t, x, y)}{p^{(\mu)}(t, x, y)} \geq 1-x^{\mu} \exp \left(\frac{x^{2}-1}{t}\right) \frac{I_{\mu}(y / t)}{I_{\mu}(x y / t)} \int_{0}^{t} q_{x}^{(\mu)}(s) d s .
$$

Since the function $z^{-\mu} I_{\mu}(z)$ is increasing on $(0, \infty)$

$$
\frac{I_{\mu}(y / t)}{I_{\mu}(x y / t)} \leq \frac{1}{x^{\mu}}, \quad x, y>1 \quad t \geq 0 .
$$

This, together with $\mathbf{P}_{x}^{(\mu)}\left(T_{1}^{(\mu)}<\infty\right)=x^{-2 \mu}$, gives

$$
\frac{p_{1}^{(\mu)}(t, x, y)}{p^{(\mu)}(t, x, y)} \geq 1-\frac{1}{x^{2 \mu}} \exp \left(\frac{x^{2}-1}{t}\right) \text {. }
$$


Now we assume that $1<x<\left(2 e^{m}\right)^{1 /(2 \mu)}$ and $t>\frac{2\left(2 e^{m}\right)^{1 / \mu}}{\mu}$. Then

$$
\frac{p_{1}^{(\mu)}(t, x, y)}{p^{(\mu)}(t, x, y)} \geq 1-\frac{1}{x^{2 \mu}} \exp \left(\frac{\mu\left(x^{2}-1\right)}{2\left(2 e^{m}\right)^{1 / \mu}}\right)
$$

The mean value theorem ensures the existence of a constant $d \in(1, x)$ such that

$$
\begin{aligned}
1-\frac{1}{x^{2 \mu}} \exp \left(\frac{\mu\left(x^{2}-1\right)}{2\left(2 e^{m}\right)^{1 / \mu}}\right) & =\frac{2 \mu(x-1)}{d^{2 \mu+1}} \exp \left(\frac{\mu\left(d^{2}-1\right)}{2\left(2 e^{m}\right)^{1 / \mu}}\right)\left(1-\frac{d^{2}}{2\left(2 e^{m}\right)^{1 / \mu}}\right) \\
& \geq c_{1}(m)(x-1),
\end{aligned}
$$

where the last inequality comes from the fact that $1<d<x<\left(2 e^{m}\right)^{1 /(2 \mu)}$.

The next step is to take $x \geq\left(2 e^{m}\right)^{(1 /(2 \mu))}$ and $t>\frac{2\left(2 e^{m}\right)^{1 / \mu}}{\mu}$. Since $x^{2}<x y<m t$ using Eq. 4.7 we get

$$
\frac{p_{1}^{(\mu)}(t, x, y)}{p^{(\mu)}(t, x, y)} \geq 1-\frac{1}{x^{2 \mu}} e^{m} \geq 1-\frac{1}{2} \approx \frac{x-1}{x} .
$$

Finally, we consider the case when $x>1, x y / m<t \leq \frac{2\left(2 e^{m}\right)^{1 / \mu}}{\mu}=: t_{0}$ and $\frac{(y-1)^{2}}{t} \geq$ $2(\mu+1)$. Using absolute continuity property (2.6) and (2.17), we can write

$$
\begin{aligned}
p_{1}^{(\mu)}(t, x, y) & \geq\left(e^{-t_{0}\left(\mu^{2} / 2-1 / 8\right)} \wedge 1\right)\left(\frac{y}{x}\right)^{\mu-1 / 2} p_{1}^{(1 / 2)}(t, x, y) \\
& \stackrel{\mu, m}{\approx}\left(1 \wedge \frac{(x-1)(y-1)}{t}\right)\left(\frac{y}{x}\right)^{\mu+1 / 2} \frac{1}{\sqrt{t}} \exp \left(-\frac{x^{2}+y^{2}}{2 t}\right) \\
& \geq\left(1 \wedge \frac{(x-1) \sqrt{2(\mu+1) / m}}{t_{0}}\right)\left(\frac{y^{2}}{t}\right)^{\mu+1 / 2} \frac{1}{\sqrt{t}} \exp \left(-\frac{x^{2}+y^{2}}{2 t}\right) \\
& \stackrel{\mu, m}{\approx} \frac{x-1}{x} p^{(\mu)}(t, x, y) .
\end{aligned}
$$

This ends the proof.

We end this section with the proof of the lower bounds, whenever $((y \vee x)-1)^{2} / t$ is small. Note that in the proof of the next proposition we use the lower bounds of $p_{1}^{(\mu)}(t, x, y)$ for $x y \geq t$ obtained previously in Section 3 as well as the result of Proposition 8. As previously, due to the symmetry, it is enough to assume that $y>x>1$.

Proposition 9 For every $\mu>0$ there exists constant $C_{9}^{(\mu)}>0$ such that

$$
\frac{p_{1}^{(\mu)}(t, x, y)}{p^{(\mu)}(t, x, y)} \geq C_{9}^{(\mu)} \frac{x-1}{x} \frac{y-1}{y}, \quad y>x>1,
$$

whenever $x y<t$ and $\frac{(y-1)^{2}}{t} \leq 2(\mu+1)$. 
Proof Let $x y<t$ and $y>x>1$. At the beginning we additionally assume that $t \geq 4$. Note that there exists $c_{1}>0$ such that for every $s>1 / 2$ we have $e^{-s} \geq c_{1} s^{\mu+1 / 2} e^{-2 s}$. This, together with the lower bounds of $p_{1}^{(\mu)}(t, z, w)$ for $z, w \geq \sqrt{t}$ (then $z w \geq t$ ) obtained in Section 3, enable us to write

$$
\begin{aligned}
p_{1}^{(\mu)}(t, z, w) & \geq c_{2}\left(1 \wedge \frac{(z-1)(w-1)}{t}\right)\left(\frac{w}{z}\right)^{\mu+1 / 2} \frac{1}{\sqrt{t}} \exp \left(-\frac{|z-w|^{2}}{2 t}\right) \\
& \geq \frac{c_{2}}{4}\left(\frac{w}{z}\right)^{\mu+1 / 2} \frac{1}{\sqrt{t}} \exp \left(-\frac{z^{2}}{2 t}\right) \exp \left(-\frac{w^{2}}{2 t}\right) \\
& \geq \frac{c_{2} c_{1}^{2}}{4}\left(\frac{w z}{t}\right)^{\mu+1 / 2}\left(\frac{w^{2}}{t}\right)^{\mu+1 / 2} \frac{1}{\sqrt{t}} \exp \left(-\frac{z^{2}}{t}\right) \exp \left(-\frac{w^{2}}{t}\right) \\
& \geq c_{3}\left(\frac{w^{2}}{t}\right)^{\mu+1 / 2} \frac{1}{\sqrt{t}} \exp \left(-\frac{z^{2}}{t}\right) \exp \left(-\frac{w^{2}}{t}\right) .
\end{aligned}
$$

Consequently, using the Chapmann-Kolmogorov equation and Eq. 2.12, we get

$$
\begin{aligned}
& p_{1}^{(\mu)}(3 t, x, y)=\int_{1}^{\infty} \int_{1}^{\infty} p_{1}^{(\mu)}(t, x, z) p_{1}^{(\mu)}(t, z, w) p_{1}^{(\mu)}(t, w, y) d z d w \\
& \geq \int_{\sqrt{t}}^{\infty} \int_{\sqrt{t}}^{\infty} p_{1}^{(\mu)}(t, x, z) p_{1}^{(\mu)}(t, z, w) p_{1}^{(\mu)}(t, w, y) d z d w \\
& \geq c_{3}\left(\frac{y^{2}}{t}\right)^{\mu+1 / 2} \frac{1}{\sqrt{t}} \int_{\sqrt{t}}^{\infty} p_{1}^{(\mu)}(t, x, z) e^{-z^{2} / t} d z \int_{\sqrt{t}}^{\infty}\left(\frac{w}{y}\right)^{2 \mu+1} p_{1}^{(\mu)}(t, w, y) e^{-w^{2} / t} d w \\
& =c_{3}\left(\frac{y^{2}}{t}\right)^{\mu+1 / 2} \frac{1}{\sqrt{t}} F_{t}^{(\mu)}(x) F_{t}^{(\mu)}(y),
\end{aligned}
$$

where

$$
F_{t}^{(\mu)}(x):=\int_{\sqrt{t}}^{\infty} p_{1}^{(\mu)}(t, x, z) e^{-z^{2} / t} d z .
$$

Since for $t \geq 4$ and $\frac{(y-1)^{2}}{t} \leq 2(\mu+1)$ we have

$$
\frac{x^{2}}{t} \leq \frac{y^{2}}{t} \leq\left(2 \wedge 4 \frac{(y-1)^{2}}{t}\right) \leq c_{4}
$$

and consequently

$$
p^{(\mu)}(3 t, x, y) \approx\left(\frac{y^{2}}{t}\right)^{\mu+1 / 2} \frac{1}{\sqrt{t}}, \quad x y<t,
$$


it is enough to show that $F_{t}^{(\mu)}(x) \geq c_{5} \frac{x-1}{x}$ for every $x>1$. However, for $z \geq b \sqrt{t}$, with $b=2 \sqrt{2(\mu+1)}$, and $t \geq 4$ we have $\frac{(z-1)^{2}}{t} \geq \frac{1}{4} \frac{z^{2}}{t} \geq 2(\mu+1)$. We can use the lower bounds given in Proposition 8 with $m=2 b$ and obtain

$$
\begin{aligned}
F_{t}^{(\mu)}(x) & \geq \int_{b \sqrt{t}}^{2 b t / x} p_{1}^{(\mu)}(t, x, z) e^{-z^{2} / t} d z \\
& \geq c_{6} \frac{x-1}{x \sqrt{t}} \int_{b \sqrt{t}}^{2 b t / x}\left(\frac{z^{2}}{t}\right)^{\mu+1 / 2} e^{-z^{2} / 2 t} e^{-z^{2} / t} d z \geq c_{7} \frac{x-1}{x \sqrt{t}} \int_{b \sqrt{t}}^{2 b t / x} e^{-2 z^{2} / t} d z \\
& =c_{7} \frac{x-1}{x} \int_{b}^{2 b \sqrt{t} / x} e^{-2 u^{2}} d u \geq c_{7} \frac{x-1}{x} \int_{b}^{2 b} e^{-2 u^{2}} d u .
\end{aligned}
$$

Finally, for $t \leq 4$, the same computations as in the end of the proof of the previous Proposition (but with $t_{0}=4$ ) gives

$$
\begin{aligned}
p_{1}^{(\mu)}(t, x, y) \geq & c_{7}\left(e^{-4\left(\mu^{2} / 2-1 / 8\right)} \wedge 1\right)\left(1 \wedge \frac{(x-1)(y-1)}{t}\right) \\
& \times\left(\frac{y^{2}}{t}\right)^{\mu+1 / 2} \frac{1}{\sqrt{t}} \exp \left(-\frac{x^{2}+y^{2}}{2 t}\right) \\
\underset{\approx}{*} & \frac{x-1}{x} \frac{y-1}{y} p^{(\mu)}(t, x, y),
\end{aligned}
$$

where the last approximation follows from the fact that $(x-1)(y-1)<x y \leq t \leq 4$ which gives

$$
1 \wedge \frac{(x-1)(y-1)}{t}=\frac{(x-1)(y-1)}{t}=\frac{(x-1)(y-1)}{x y} \frac{x y}{t} \approx \frac{(x-1)(y-1)}{x y} .
$$

Open Access This article is distributed under the terms of the Creative Commons Attribution License which permits any use, distribution, and reproduction in any medium, provided the original author(s) and the source are credited.

Acknowledgments The authors are very grateful to Tomasz Byczkowski for critical remarks and comments which enabled them to improve the presentation of the paper.

\section{References}

1. Borodin, A.N., Salminen, P.: Handbook of Brownian Motion - Facts and Formulae, 2nd edn. Birkhauser Verlag, Basel (2002)

2. Byczkowski, T., Graczyk, P., Stos, A.: Poisson kernels of half-spaces in real hyperbolic spaces. Rev. Mat. Iberoamericana 23(1), 85-126 (2007)

3. Byczkowski, T., Małecki, J., Ryznar, M.: Hitting times of Bessel processes. Potential Anal. 38, 753-786 (2013)

4. Byczkowski, T., Ryznar, M.: Hitting distibution of geometric Brownian motion. Studia Math. 173(1), 19-38 (2006)

5. Ciaurri, Ó., Roncal, L.: The Bochner-Riesz means for Fourier-Bessel expansions. J. Funct. Anal. 228, 89-113 (2005)

6. Ciaurri, Ó., Roncal, L.: Littlewood-Paley-Stein $g_{k}$-functions for Fourier-Bessel expansions. J. Funct. Anal. 258, 2173-2204 (2010)

7. Ciaurri, Ó., Stempak, K.: Conjugacy for Fourier-Bessel expansions. Studia Math. 176, 215-247 (2006) 
8. Ciaurri, Ó., Stempak, K.: Transplantation and multiplier theorems for Fourier-Bessel expansions. Trans. Amer. Math. Soc. 358, 4441-4465 (2006)

9. Ciaurri, Ó., Stempak, K.: Weighted transplantation for Fourier-Bessel expansions. J. Anal. Math. 100, 133-156 (2006)

10. Davies, E.B.: The equivalence of certain heat kernel and Green function bounds. J. Funct. Anal. 71, 88-103 (1987)

11. Davies, E.B.: Heat kernels and spectral theory (Cambridge Tracts in Mathematics)volume 92. Cambridge University Press, Cambridge (1990)

12. Davies, E.B.: Intrinsic ultracontractivity and the dirichlet laplacian. J. Funct. Anal. 100, 162-180 (1991)

13. Davies, E.B., Simon, B.: Ultracontractivity and heat kernels for Schrdinger operators and Dirichlet Laplacians. J. Funct. Anal. 59, 335-395 (1984)

14. Erdelyi et al.: Higher Transcendental Functions, vol. II. McGraw-Hill, New York (1953)

15. Evans, L.C.: Partial differential equations. Amer. Math. Soc. (1998)

16. Gradstein, I.S., Ryzhik, I.M.: Table of integrals, series and products, 7 th edn. Academic Press, London (2007)

17. Gyrya, P., Saloff-Coste, L.: Neumann and Dirichlet heat kernels in inner uniform domains. Astérisque (2011)

18. Hamana, Y., Matsumoto, H.: Hitting times of Bessel processes, volume of Wiener sausages and zeros of Macdonald functions arXiv:1302.4526

19. Hamana, Y., Matsumoto, H.: The probability densities of the first hitting times of Bessel processes. J. Math-for-Ind. 4B, 91-95 (2012)

20. Hamana, Y., Matsumoto, H.: The probability distributions of the first hitting times of Bessel processes. Trans. Amer. Math. Soc. 365, 5237-5257 (2013)

21. Laforgia, A.: Bounds for modified Bessel functions. J. Comput. Appl. Math. 34, 263-267 (1991)

22. Matsumoto, H., Yor, M.: Exponential functionals of Brownian motion, I: Probability laws at fixed time. Probability Surveys 2, 312-347 (2005)

23. Matsumoto, H., Yor, M.: Exponential functionals of Brownian motion, II: Some related diffusion processes. Probability Surveys 2, 348-384 (2005)

24. Nowak, A., Roncal, L.: On sharp heat and subordinated kernel estimates in the Fourier-Bessel setting. Rocky Mountain J. Math. 44, 1321-1342 (2014)

25. Nowak, A., Roncal, L.: Sharp heat kernel estimates in the Fourier-Bessel setting for a continuous range of the type parameter. Acta Math. Sin. (Engl. Ser.) 30, 437-444 (2014)

26. Saloff-Coste, L.: The heat kernel and its estimates. Adv. Stud. Pure Math. 57, 405-436 (2010)

27. Zhang, Q.S.: The boundary behavior of heat kernels of Dirichlet Laplacians. J. Differential Equations 182, 416-430 (2002) 